\section{Effect of Celliant $\AA$ armbands on grip strength in subjects with chronic wrist and elbow pain: randomized double-blind placebo-controlled trial}

\author{
Ian L. Gordon
}

Veterans Affairs Long Beach Healthcare System, Long Beach, California, USA

Seth Casden

Hologenix, Pacific Pallisades, California, USA, and

Michael R. Hamblin

Laser Research Center, University of Johannesburg - Doornfontein Campus, Doornfontein, South Africa
Chronic wrist and elbow pain

Received 16 March 2021 Revised 26 April 2021 Accepted 29 May 2021

\begin{abstract}
Purpose - This study aims to test the effects of Celliant armbands on grip strength in subjects with chronic wrist and elbow pain. Celliant $\mathbb{R}$ is a functional textile fabric containing minerals that emit infrared radiation (IR) in response to body heat. IR-emitting fabrics have biological effects including the reduction of pain and inflammation and the stimulation of muscle function.
\end{abstract}

Design/methodology/approach - A randomized placebo-controlled trial recruited 80 subjects (40 per group) with a six-month history of chronic wrist or elbow pain (carpal tunnel syndrome, epicondylitis or arthritis) to wear an armband (real Celliant or placebo fabric) on the affected wrist or elbow for two weeks. Grip strength was measured by a dynamometer before and after the two-week study.

Findings - For the placebo group, the mean grip strength increased from $47.95 \pm 25.14$ (baseline) to $51.69 \pm 27.35$ (final), whereas for the Celliant group, it increased from $46.3 \pm 22.02$ to $54.1 \pm 25.97$. The mean per cent increase over the two weeks was $+7.8 \%$ for placebo and $+16.8 \%$ for Celliant $(p=0.0372)$. No adverse effects was observed.

Research limitations/implications - Limitations include the wide variation in grip strength in the participants at baseline measurement, which meant that only the percentage increase between baseline and final measurements showed a significant difference. Moreover, no subjective measurements of pain or objective neurophysiology testes was done.

Practical implications - Celliant armbands are easy to wear and have not been shown to produce any adverse effects. Therefore, there appears to be no barrier to prevent widespread uptake.

Social implications - IR-emitting textiles have been studied for their beneficial effects, both in patients diagnosed with various disorders and also in healthy volunteers for health and wellness purposes. Although there are many types of textile technology that might be used to produce IR-emitting fabrics, including coating of the fabric with a printed layer of ceramic material, incorporating discs of mineral into the garment,

(C) Ian L. Gordon, Seth Casden and Michael R. Hamblin. Published by Emerald Publishing Limited. This article is published under the Creative Commons Attribution (CC BY 4.0) license. Anyone may reproduce, distribute, translate and create derivative works of this article (for both commercial and non-commercial purposes), subject to full attribution to the original publication and authors. The full terms of this license may be seen at http://creativecommons.org/licences/by/4.0/legalcode
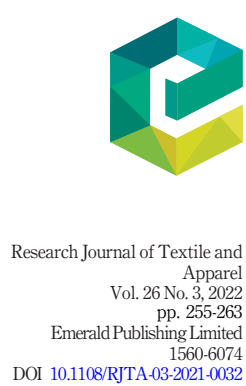
RJTA

26,3

the authors feel that incorporating ceramic particles into the polymer fibers from which the fabric is woven is likely to be the most efficient way of achieving the goal.

Originality/value - Celliant armbands appear to be effective in painful upper limb inflammatory disorders, and further studies are warranted. The mechanism of action is not completely understood, but the hypothesis that the emitted IR radiation is absorbed by nanostructured intracellular water provides some theoretical justification.

Keywords Fabric, Textile, Thermal, Radiant protective performance, Placebo-controlled clinical trial, Infrared-emitting fabric, Functional textile, Chronic wrist and elbow pain, Grip strength

Paper type Research paper

\section{Introduction}

The biological effects of infrared radiation (IR) $(3-100 \mu \mathrm{m})$ that have been observed in both cellular and animal studies in the laboratory, and also in human studies in vivo, have suggested that IR could be a promising therapeutic modality to treat certain medical conditions (reviewed in Vatansever and Hamblin, 2012). Technological advances have provided new routes to deliver IR radiation to the human body. Specialized lamps (IR heat lamps) and IR saunas can deliver pure IR radiation and are now safe, attractive and widely used devices to generate therapeutic effects.

When IR is used as a therapeutic modality, the alternative terms "biogenetic radiation" and "biogenetic rays" have been used in the popular literature. The IR wavelength is too long to be perceived by the human eye, however, the body experiences its energy as a gentle radiant heat which can penetrate over 1.5 inches $(4 \mathrm{~cm})$ beneath the skin (Yu et al., 2006). In the IR radiation bands, wavelengths between 3 and $20 \mu \mathrm{m}$ transfer electromagnetic energy that can be perceived by the thermoreceptors in the human skin as radiant heat (Sheppard et al., 2008). This is principally because these wavelengths are absorbed by water molecules in cells and tissues leading to an increase in the molecular vibrations. Not only is IR absorbed by the human body, but it is also emitted by the body in the form of blackbody radiation (3 to 50 microns, with an output peak at 9.4 micron). IR energy is sufficient to increase the rotational and vibrational modes of bond motion in biological molecules (including water molecules). The resulting epidermal temperature is higher when the skin is irradiated with IR than if similar energy densities delivered using shorter visible wavelengths are used. The prolonged erythermal response from IR exposure has been proposed to be because of increasing the epidermal temperature, but levels of IR that do not produce any detectable skin heating can also have biological effects.

In addition to the use of IR heat lamps and IR saunas, there is another technological solution to the problem of delivering IR radiation to the human body. This involves the use of specific minerals that are able to absorb the heat produced by the body, in the form of conduction, convection or radiation and reemit the energy back into the body in the form of IR with a broad peak centered at $9.4 \mu \mathrm{m}$. Although some approaches have used discs or powders made of minerals such as jade (Yoo et al., 2002) or sericite (Lee et al., 2011), that are held in contact with the body, a more realistic approach is to incorporate micron-sized particles of IR-emitting minerals into polymer fibers from which fabrics can be woven. These functional textiles can be made into garments, bandages or bed sheets that can be worn or maintained in contact with the body for many hours, as opposed to the relatively brief periods possible with lamps or saunas (Vatansever and Hamblin, 2012). As might be expected, the power density of IR-emitting textiles, which are solely powered by body-heat is rather low, when compared with lamps or saunas which are electrically powered. 
However, this low power density is compensated by the longer time that it can be maintained in contact with the body.

IR-emitting textiles have been studied for their beneficial effects, both in patients diagnosed with various disorders and also in healthy volunteers for health and wellness purposes.

\section{Materials and methods}

Celliant and placebo wrist and elbow bands

Celliant ${ }^{\circledR}$ technology is a patented process for adding micron-sized thermo-responsive quartz, silicon oxide and titanium oxide particles to polyethylene terephalate (PET) fibers. The resulting Celliant ${ }^{\circledR}$ yarns were woven into armbands containing either $42 \%$ Celliant PET (active) or zero Celliant PET (placebo). They were produced using a seamless construction with either type of yarn. The two armbands are shown in Figure 1.

\section{Study design}

This was a single center, double-blind, randomized, placebo-controlled, parallel group comparison study of arm and wrist bands constructed from Celliant versus placebo fabric in subjects with chronic upper extremity pain. The protocol was approved by the IRB of Southern California Institute for Research and Education and registered at clinicaltrials.gov NCT00688220. The protocol was in accordance with the Declaration of Helsinki, and verbal informed consent was obtained. The inclusion criteria were, both males and females, aged 21 or older, presence of an inflammatory condition causing pain for a minimum of six months affecting the elbow, forearm or wrist such as carpal tunnel syndrome or lateral epicondylitis. The exclusion criteria were any individual who received increased or decreased pain or analgesic medication during the study, the presence of substance abuse or psychiatric

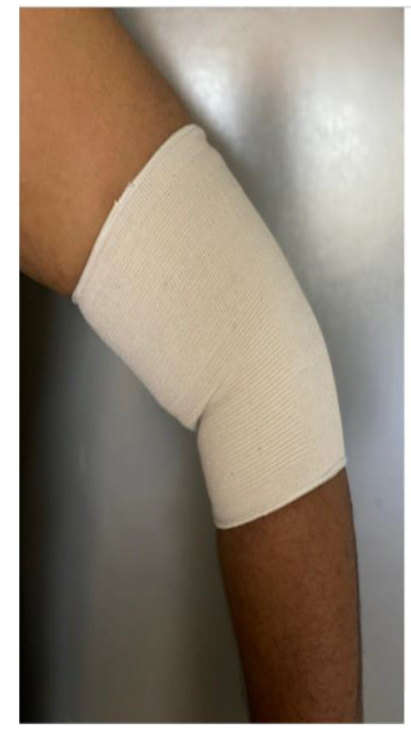

(a)

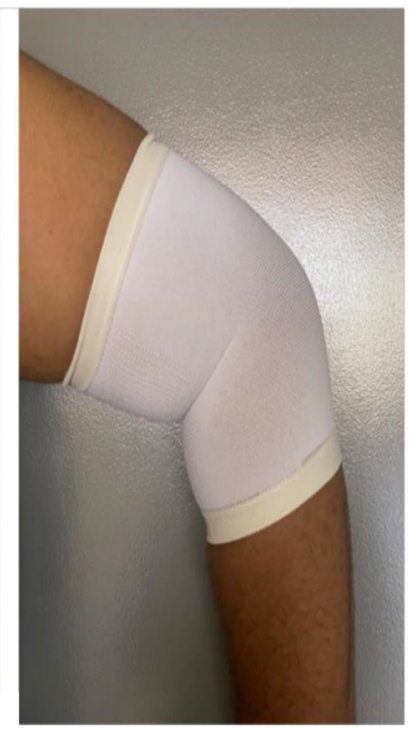

(b)
Figure 1.

(a) Placebo and (b) Celliant elbow sleeves 
RJTA

26,3

258

conditions, which interfered with the subject complying with the study protocol, open wounds on the involved limb and threatened limb loss from ischemia.

\section{Intervention}

The grip strength expressed as pounds of force was measured using the Baseline Hydraulic Hand Dynamometer manufactured by Fabrication Enterprises Inc. (White Plains, NY). Participants were tested at baseline for grip strength in the painful hand using three repetitive tests $5 \mathrm{~min}$ apart. Participants were then randomized to receive either a real Celliant or placebo PET armband (Figure 1) and were instructed to wear it on the painful elbow or wrist for at least $12 \mathrm{~h}$ per day for 14 days. At the completion of the 14 days period, participants returned for a repeat of the three repetitions of the dynamometer test and to report any adverse effects.

\section{Statistical analysis}

Values are presented as means and standard deviations. Comparison of means was carried out using a two-tailed unpaired student's $t$-test in GraphPad (available at: www.graphpad. com/quickcalcs/ttest1.cfm). $P$ values $<0.05$ were considered significant.

\section{Results}

There were 12 dropouts out of the initial 80 participants, so that in the placebo group 33 participants completed the trial, and in the Celliant group, 35 participants completed the trial. There were no adverse effects reported. The mean values of the three repetitive grip strength tests taken at each visit and the maximum strength value out of the three repetitions for placebo and Celliant at baseline and at 2 weeks are shown in Table 1 . There was a large variation between individuals in the trial in terms of grip strength, so that the standard deviations were relatively high. For instance, the range of grip strength at the baseline measurement was 14-105 pounds force for the placebo group and 10-110 pounds force for the Celliant group. Likewise the range at the final measurement was 13-120 pounds force for the placebo group and 13-100 pounds force for the Celliant group. This wide variation meant that there was no statistically significant differences in the mean grip strength between before and after measurements in either group. However when the mean per cent increase in grip strength between baseline and final measurements was calculated for each individual, the mean values of these increases was $7.8 \pm 21.7 \%$ for the placebo group and $16.8 \pm 26.93 \%$ for the Celliant group. These values were significantly different with $p=0.0372$ by a two-tailed unpaired student's $t$-test (Figure 2).

\section{Discussion}

A possible explanation for the biological effects that occur in tissue exposed to IRemitting fabrics without any detectable temperature change, is that the IR radiation is

Table 1.

Mean and maximum grip strength (pounds force) at initial and final measurements for placebo and Celliant arm bands

\begin{tabular}{lcccc}
\hline & Initial placebo & Final placebo & Initial Celliant & Final Celliant \\
\hline Number completed & & 33 & & 35 \\
Mean strength & $47.95 \pm 25.14$ & $51.69 \pm 27.35$ & $46.3 \pm 22.02$ & $54.1 \pm 25.97$ \\
Maximum strength & $53.33 \pm 26.07$ & $56.84 \pm 28.16$ & $51.53 \pm 24.54$ & $59.5 \pm 28.07$ \\
Mean \% increase mean strength & & $7.8 \pm 21.7$ & & $16.8 \pm 26.93^{*}$ \\
Note: ${ }^{*} p<0.05$ Celliant vs placebo & & & \\
& & & \\
\end{tabular}


selectively absorbed by water molecules that are associated with ion channels within the cell membranes ( $\mathrm{Yu}$ et al., 2006). Water is by far the most important tissue chromophore in the IR spectral range, both because of its high absorption coefficient and its high abundance in tissue. These water molecules are absorbed on the surface of the so-called "heat-sensitive transient receptor potential (TRP) ion channels." When these "structured" water molecules absorb IR, they change their $\mathrm{pH}$ value by a small but significant amount, which affects the conformation of critical amino acids in the protein components of the ion channel. These TRP channels are calcium ion channels and elbow pain and the resulting increase in cellular calcium can activate several important signaling pathways and even activate transcription factors. One specific TRP channel (TRPV3) has been linked to epithelial wound healing (Aijima et al., 2015) through inducing the release of nitric oxide (NO) from keratinocytes (Cals-Grierson and Ormerod, 2004, Miyamoto et al., 2011). Another TRP channel, TRPC6 has been linked to the regulation of fibroblast-myofibroblast transdifferentiation, and deletion of this protein in the laboratory resulted in delayed dermal wound healing in rodents (Davis et al., 2012).

There is a growing body of evidence describing the use of garments and wraps designed to be worn for extended periods of time and to deliver effective doses of IR radiation into the underlying tissue. Fibers impregnated with various mineralcontaining materials can then be woven into fabrics, which can then be worn as garments or wraps and used to provide health benefits to the wearer. In a similar manner, discs of IR-emitting ceramic material have also been applied to the human body to produce a beneficial effect. For instance, a blanket containing mineral discs was reported to improve the quality of sleep (Kotorii et al., 1988), and single discs were applied to the breasts of women who encountered difficulty in producing sufficient breast milk during lactation (Ogita et al., 1990). McCall et al. (2018) reported a clinical trial using bedsheets woven from IR-emitting fabrics and found that participants randomized to sleep with active bedsheets reported fewer insomnia symptoms compared to those with control sheets. Gloves have been fabricated from IR-emitting fabrics, and there have been reports that these gloves can be used to treat arthritis of the hands and Raynaud's syndrome (Ko and Berbrayer, 2002). Mantegazza et al. (2018) conducted a double-blind, crossover trial in healthy volunteers, where athletic outfits woven from IR-emitting fabric or control fabric were worn while completing a maximal

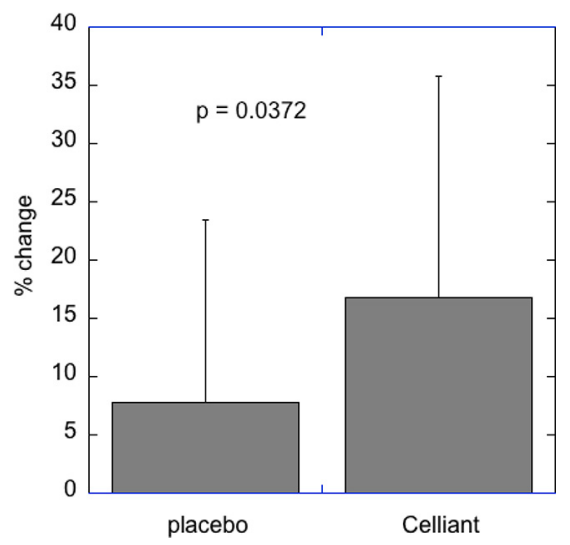

Figure 2. Mean $\%$ increase of final grip strength over baseline grip strength for subjects wearing placebo $(n=33)$ or Celliant $(n=35)$ armbands daily for two weeks. Error bars are SD 
RJTA

26,3

cardiopulmonary exercise test. Several parameters were significantly improved, including peak oxygen uptake endurance time, anaerobic threshold and blood lactate concentration. Sakugawa et al. (2020) studied patients suffering from lower leg edema, who wore socks woven from IR-emitting fabric or control for $8 \mathrm{~h} /$ day for 28 days. The active group showed significantly less swelling and lower pain scores compared to the control group.

Celliant ${ }^{\circledR}$ fibers, yarns and fabrics, such as other products described above, are designed to reemit the heat energy generated by the wearer in the form of infrared light back into the wearer's skin (Vatansever and Hamblin, 2012).

A recent laboratory study (Djuretić et al., 2021) confirmed that Celliant fibers containing ceramic particles had beneficial effects in a rat model of collagen type II-induced arthritis (CIA). Rats were housed in cages with bedding composed either of Celliant fibers or standard wooden shavings. The appearance of the symptoms of CIA was delayed, whereas the disease was milder (judging by the arthritis score, paw volume and burrowing behavior) in Celliant-treated rats as compared to controls. This correlated with lower values of serum anti-CII IgG antibody levels measured in Celliant rats and lower levels of IL-17 in cell cultures produced from their paws. Additionally, in rats with carrageenan-induced paw inflammation (CIPI), IR from Celliant fibers exerted an anti-inflammatory effect and improved burrowing behavior in CIPI rats.

There have been three clinical trials published so far that have reported the application of Celliant-containing fabrics or garments for various purposes. York and Gordon (York and Gordon, 2009) recruited 55 patients with foot pain (diabetic neuropathy or other causes) to wear either placebo or Celliant socks for two weeks. Wearers of Celliant socks reported significantly less pain compared to placebo socks.

Washington et al. (2018) recruited 153 volunteers to wear either a placebo or a Celliant shirt in different orders of donning with a $90 \mathrm{~min}$ interval between them. Independent of the donning sequence, the transcutaneous blood oxygenation (tcPO2) measurements were significantly higher after wearing a Celliant shirt compared to a placebo shirt.

Gordon et al. (2019) carried out a follow-up study on 24 volunteers. The participants first wore a placebo shirt for $90 \mathrm{~min}$ and then a Celliant shirt for $90 \mathrm{~min}$. The mean tcPO2 measured at two sites (biceps and abdomen) was significantly higher at three time points (30,60 and $90 \mathrm{~min})$ in Celliant vs placebo. The mean grip strength in the dominant hand measured at 90 min was $12.44 \%$ higher after wearing Celliant vs placebo $(p=0.0002)$.

The present study has added yet another example of the beneficial effects of Celliant fabric, this time worn as an armband in subjects with chronic pain conditions because of carpal tunnel syndrome or to lateral epicondylitis. Both conditions, in addition to being painful, are well-known to reduce grip strength (Sasaki et al., 2020, Kim et al., 2020). There are a variety of treatments for these conditions (Kim et al., 2019, Li et al., 2020), but none are completely effective in these chronic conditions. The improvement in the grip strength observed in the present study was in agreement with the improvement in grip strength seen in our previous study (Gordon et al., 2019), but in that case volunteers who were healthy without any disease wore shirts woven from either Celliant fabric or control fabric, and the grip strength in the dominant hand was measure to be higher, although the hands were not in contact with the actual fabric. This finding suggests that there is a systemic effect of Celliant fabric in addition to any local effects.

The noninvasive nature and the cost-effectiveness of daily wearing of Celliant armbands suggests that this approach should be further investigated in clinical trials with the addition of objective measurements of pain and neurophysiology. 


\section{Competing interests}

The authors declare the following conflicts of interest. SG is a stockholder and employee of Hologenix LLC that manufacturers Celliant fabric, and which funded this study. ILG received sponsored research funding from Hologenix LLC. MRH received consulting fees and is on the scientific advisory board of Hologenix LLC. MRH declares the additional possible conflicts of interest. Scientific Advisory Boards: Transdermal Cap Inc, Cleveland, $\mathrm{OH}$; BeWell Global Inc, Wan Chai, Hong Kong; LumiThera Inc, Poulsbo, WA; Vielight, Toronto, Canada; Bright Photomedicine, Sao Paulo, Brazil; Quantum Dynamics LLC, Cambridge, MA; Global Photon Inc, Bee Cave, TX; Medical Coherence, Boston MA; NeuroThera, Newark DE; JOOVV Inc, Minneapolis-St. Paul MN; AIRx Medical, Pleasanton CA; FIR Industries, Inc. Ramsey, NJ; UVLRx Therapeutics, Oldsmar, FL; Ultralux UV Inc, Lansing MI; Illumiheal \& Petthera, Shoreline, WA; MB Lasertherapy, Houston, TX; ARRC LED, San Clemente, CA; Varuna Biomedical Corp. Incline Village, NV; Niraxx Light Therapeutics, Inc, Boston, MA. Consulting; Lexington Int, Boca Raton, FL; USHIO Corp, Japan; Merck KGaA, Darmstadt, Germany; Philips Electronics Nederland B.V. Eindhoven, Netherlands; Johnson \& Johnson Inc, Philadelphia, PA; Sanofi-Aventis Deutschland GmbH, Frankfurt am Main, Germany. Stockholdings: Global Photon Inc, Bee Cave, TX; Mitonix, Newark, DE.

\section{Authors' contributions}

ILG participated in the conception and design of the study, carried out subject recruitment and data collection, took part in the statistical analysis. SC participated in the conception and design of the study, provided materials and funding, and helped draft the manuscript. MRH wrote the manuscript and critically reviewed the data. All authors read and approved the final manuscript.

\section{Funding}

The study was funded by Hologenix LLC. MRH was funded by US NIH Grants R01AI050875 and R21AI121700.

\section{References}

Aijima, R., Wang, B., Takao, T., Mihara, H., Kashio, M., Ohsaki, Y., Zhang, J.Q., Mizuno, A., Suzuki, M., Yamashita, Y., Masuko, S., Goto, M., Tominaga, M. and Kido, M.A. (2015), "The thermosensitive TRPV3 channel contributes to rapid wound healing in oral epithelia", The Faseb Journal, Vol. 29 No. 1, pp. 182-192.

Cals-Grierson, M.M. and Ormerod, A.D. (2004), "Nitric oxide function in the skin", Nitric Oxide, Vol. 10 No. 4, pp. 179-193.

Davis, J., Burr, A.R., Davis, G.F., Birnbaumer, L. and Molkentin, J.D. (2012), “A TRPC6-dependent pathway for myofibroblast transdifferentiation and wound healing in vivo", Developmental Cell, Vol. 23 No. 4, pp. 705-715.

Djuretić, J., Dimitrijević, M., Stojanović, M., Stevuljević, J.K., Hamblin, M.R., Micov, A., StepanovićPetrović, R. and Leposavić, G. (2021), "Infrared radiation from cage bedding moderates rat inflammatory and autoimmune responses in collagen-induced arthritis", Scientific Reports, Vol. 11 No. 1.

Gordon, I.L., Casden, S., Vangel, M. and Hamblin, M.R. (2019), "Effect of shirts with $42 \%$ celliant fiber on tcPO2 levels and grip strength in healthy subjects: a placebo-controlled clinical trial", J Textile Sci Eng, Vol. 9 No. 4, pp. 1000403 
RJTA

26,3

Kim, Y.J., Wood, S.M., Yoon, A.P., Howard, J.C., Yang, L.Y. and Chung, K.C. (2020), "Efficacy of nonoperative treatments for lateral epicondylitis: a systematic review and Meta-Analysis", Plastic and Reconstructive Surgery, Vol. 147 No. 1, pp. 112-125.

Kim, G.M., Yoo, S.J., Choi, S. and Park, Y.-G. (2019), "Current trends for treating lateral epicondylitis", Clinics in Shoulder and Elbow, Vol. 22 No. 4, pp. 227-234.

Ko, G.D. and Berbrayer, D. (2002), "Effect of ceramic-impregnated thermoflow gloves on patients with raynaud's syndrome: randomized, placebo-controlled study", Alternative Medicine Review: a Journal of Clinical Therapeutic, Vol. 7 No. 4, pp. 328-335.

Kotorii, T., Nonaka, K., Hayashida, N., Miyahara, Y., Ohse, K. and Nakazawa, Y. (1988), "Effects of farinfrared radiation on sleep and body temperature in healthy adults", Kyushu Neuro-Psychiat, Vol. 34, pp. 63-67.

Lee, C.H., Roh, J.W., Lim, C.Y., Hong, J.H., Lee, J.K. and Min, E.G. (2011), “A multicenter, randomized, double-blind, placebo-controlled trial evaluating the efficacy and safety of a far infrared-emitting sericite belt in patients with primary dysmenorrhea", Complementary Therapies in Medicine, Vol. 19 No. 4, pp. 187-193.

Li, W., Dong, C., Wei, H., Xiong, Z., Zhang, L., Zhou, J., Wang, Y., Song, J. and Tan, M. (2020), "Extracorporeal shock wave therapy versus local corticosteroid injection for the treatment of carpal tunnel syndrome: a Meta-analysis", Journal of Orthopaedic Surgery and Research, Vol. 15 No. 1.

McCall, W.V., Letton, A., Lundeen, J., Case, D. and Cidral-Filho, F.J. (2018), "The effect of farinfrared emitting sheets on sleep", Research Journal of Textile and Apparel, Vol. 22 No. 3, pp. 247-259.

Mantegazza, V., Contini, M., Botti, M., Ferri, A., Dotti, F., Berardi, P. and Agostoni, P. (2018), "Improvement in exercise capacity and delayed anaerobic metabolism induced by far-infraredemitting garments in active healthy subjects: a pilot study", European Journal of Preventive Cardiology, Vol. 25 No. 16, pp. 1744-1751.

Miyamoto, T., Petrus, M.J., Dubin, A.E. and Patapoutian, A. (2011), "TRPV3 regulates nitric oxide synthase-independent nitric oxide synthesis in the skin", Nature Communications, Vol. 2 No. 1, p. 369.

Ogita, S., Imanaka, M., Matsuo, S., Takebayashi, T., Nakai, Y., Fukumasu, H., Matsumoto, M. and Iwanaga, K. (1990), "Effects of far-infrared radiation on lactation", The Annals of Physiological Anthropology, Vol. 9 No. 2, pp. 83-91.

Sakugawa, A.A.D.S., Conrado, L.A.L., Villaverde, A.B. and Munin, E. (2020), “Antiedematous effect promoted by occlusion of legs with compressive socks containing InfraredEmitting ceramic particulates", Photobiomodulation, Photomedicine, and Laser Surgery, Vol. 38 No. 1, pp. 51-56.

Sasaki, T., Makino, K., Nimura, A., Suzuki, S., Kuroiwa, T., Koyama, T., Okawa, A., Terada, H. and Fujita, K. (2020), "Assessment of grip-motion characteristics in carpal tunnel syndrome patients using a novel finger grip dynamometer system", Journal of Orthopaedic Surgery and Research, Vol. 15 No. 1.

Sheppard, A.R., Swicord, M.L. and Balzano, Q. (2008), "Quantitative evaluations of mechanisms of radiofrequency interactions with biological molecules and processes", Health Physics, Vol. 95 No. 4, pp. 365-396.

Vatansever, F. and Hamblin, M.R. (2012), "Far infrared radiation (FIR): its biological effects and medical applications", Photonics Lasers Med, Vol. 4, pp. 255-266.

Washington, K., Wason, J., Thein, M.S., Lavery, L.A., Hamblin, M.R. and Gordon, I.L. (2018), "Randomized controlled trial comparing the effects of Far-Infrared emitting ceramic fabric shirts and control polyester shirts on transcutaneous PO2", Journal of Text Sci Eng, Vol. 8 No. 2. 
Yoo, B.H., Park, C.M., Oh, T.J., Han, S.H., Kang, H.H. and Chang, I.S. (2002), "Investigation of jewelry powders radiating far-infrared rays and the biological effects on human skin", Journal of Cosmet Sci, Vol. 53 No. 3, pp. 175-184.

Chronic wrist and elbow pain

York, R.M. and Gordon, I.L. (2009), "Effect of optically modified polyethylene terephthalate fiber socks on chronic foot pain", BMC Complementary and Alternative Medicine, Vol. 9 No. 1, p. 10.

Yu, S.Y., Chiu, J.H., Yang, S.D., Hsu, Y.C., Lui, W.Y. and Wu, C.W. (2006), "Biological effect of far-infrared therapy on increasing skin microcirculation in rats", Photodermatology, Photoimmunology and Photomedicine, Vol. 22 No. 2, pp. 78-86.

Corresponding author

Michael Hamblin can be contacted at: hamblin.lab@gmail.com

For instructions on how to order reprints of this article, please visit our website: www.emeraldgrouppublishing.com/licensing/reprints.htm Or contact us for further details: permissions@emeraldinsight.com 